\title{
Proton Conductivity of Metallo-Supramolecular Polymer Boosted by Lithium Ions
}

Yemineni S L V Narayana, Takefumi Yoshida, Chanchal Chakraborty, Manas Kumar Bera, and Masayoshi Higuchi*

Electronic Functional Macromolecules Group, National Institute for Materials Science (NIMS), Tsukuba 305-0044, Japan, Email: HIGUCHI.Masayoshi@nims.go.jp 


\section{Contents}

1. Characterization of polymers using NMR and FT-IR spectroscopy

2. XPS Spectra of polymers

3. Thermogravimetric analysis of polymers before and after the impedance measurements

4. Powder -XRD Study of polymers and ligand molecule

5. Impedance measurement of polymers using the in-plane experimental method

6. Conductivity electrode (Platinum) used for the in-plane experiment

7. Impedance measurement of polyEu- $\mathrm{Li}^{+}$using the thick pellet

8. Impedance measurement of polymers at different humidity condition

9. Log conductivity vs log frequency plots of polymers for through plane, in-plane and thick films data.

10. PL Spectra of polymers at ambient and $95 \%$ RH condition

11. Through -plane and in-plane proton conductivities of polymers at $95 \% \mathrm{RH}$ and $25^{\circ} \mathrm{C}$

12. Comparative proton conductivities of polymers at different humidity 
1. Characterization of the polymers using NMR and FT-IR

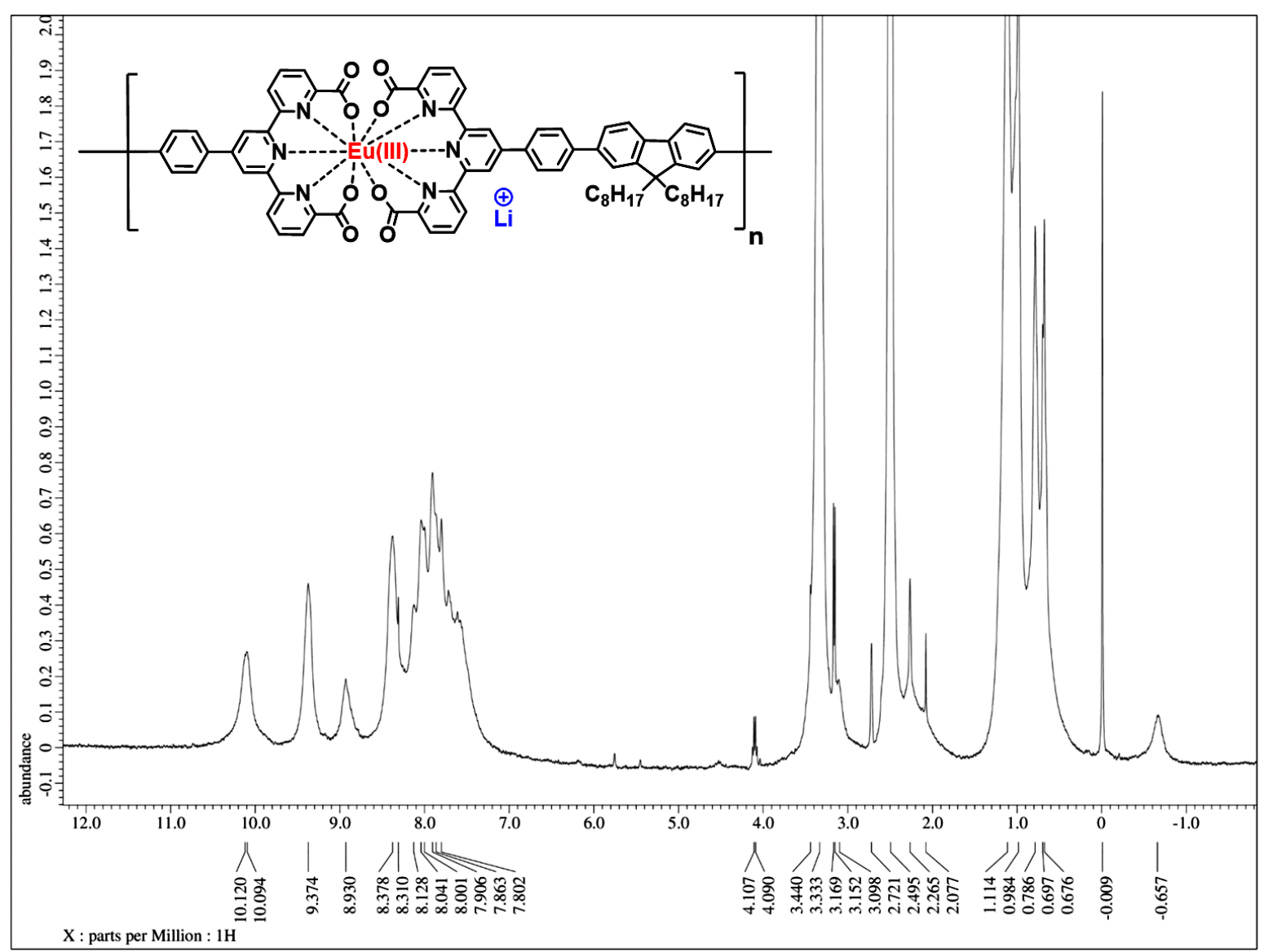

Figure S1. ${ }^{1} \mathrm{H}$ - NMR spectrum of polyEu-Li ${ }^{+}$. 


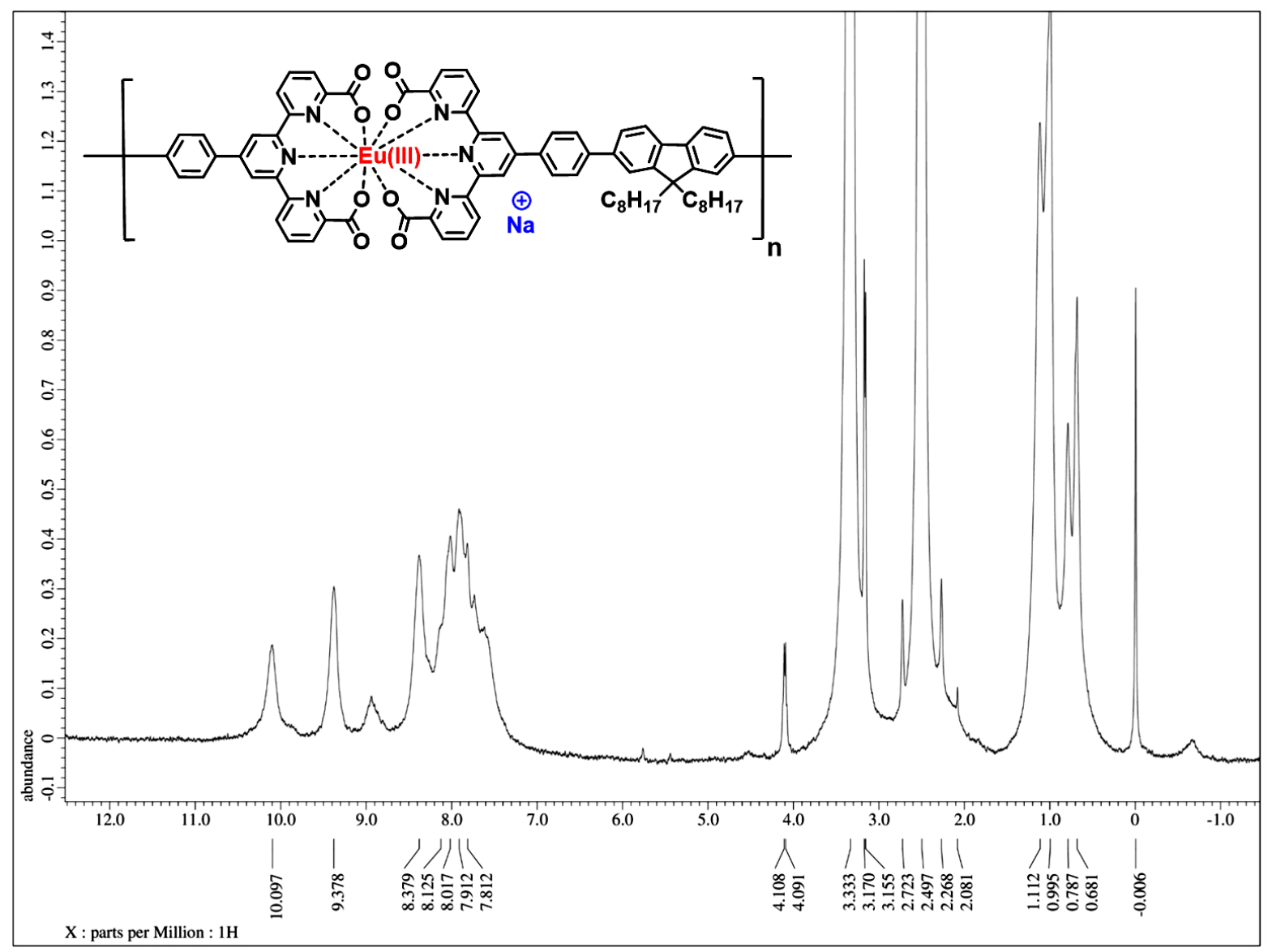

Figure S2. ${ }^{1} \mathrm{H}-\mathrm{NMR}$ spectrum of polyEu-Na+ 


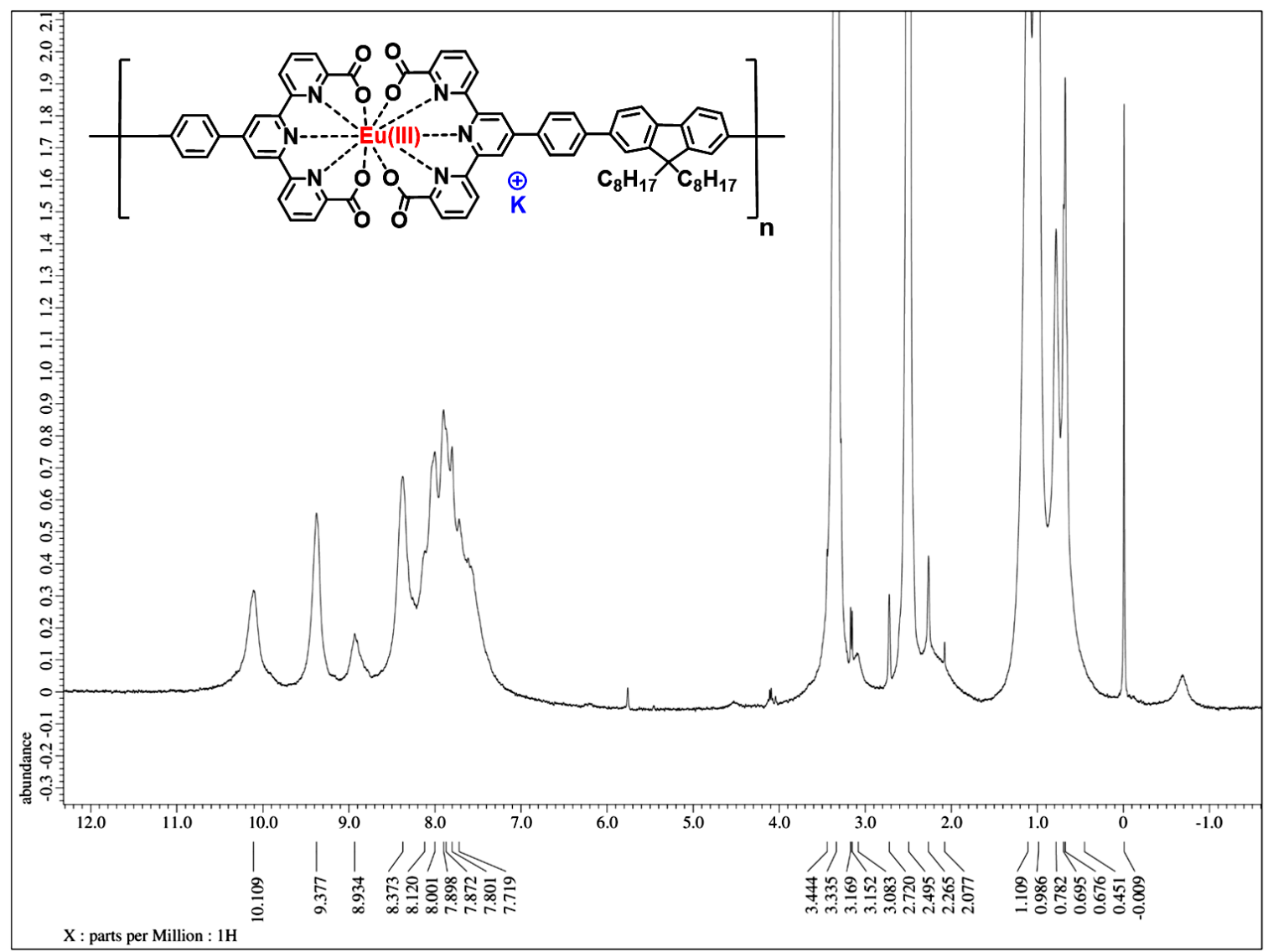

Figure S3. ${ }^{1} \mathrm{H}-\mathrm{NMR}$ spectrum of polyEu-K ${ }^{+}$ 
(a)

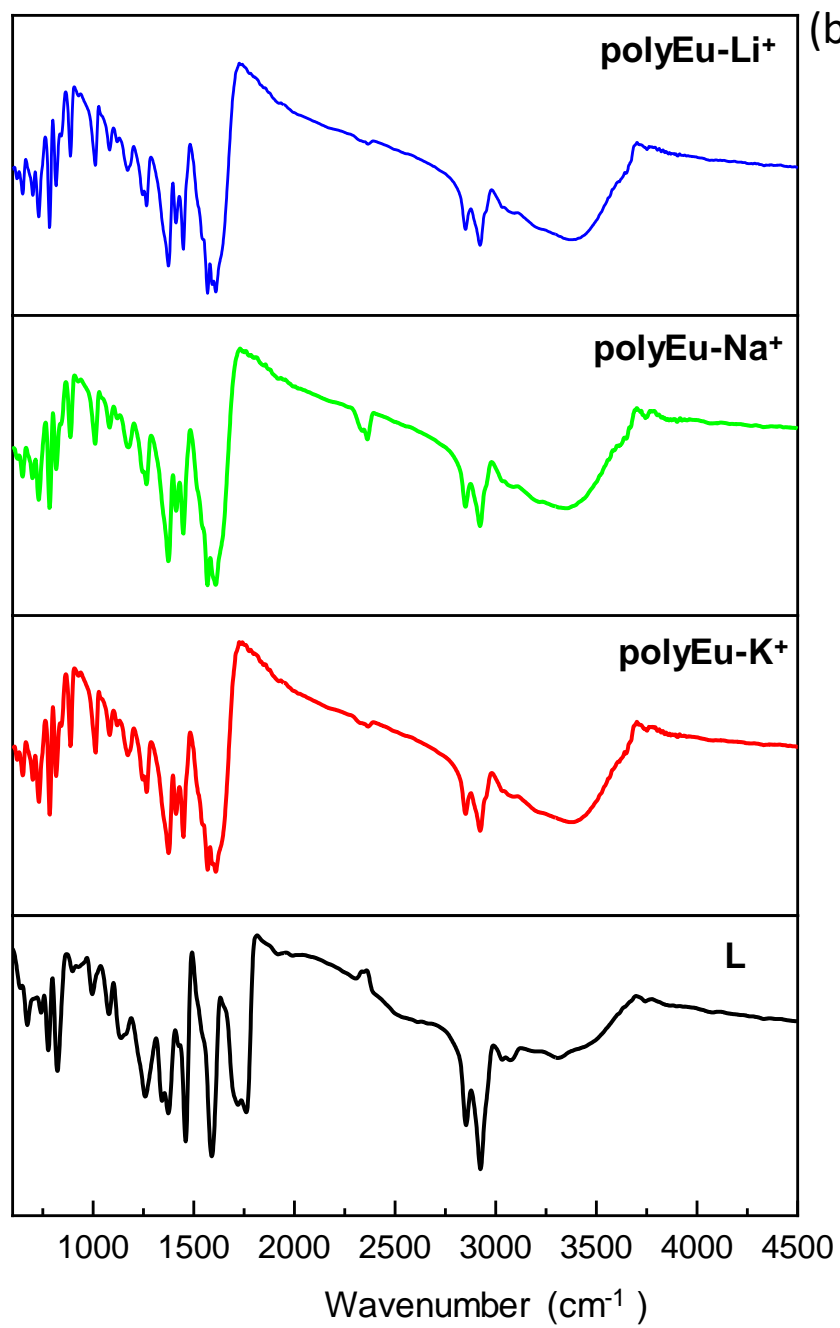

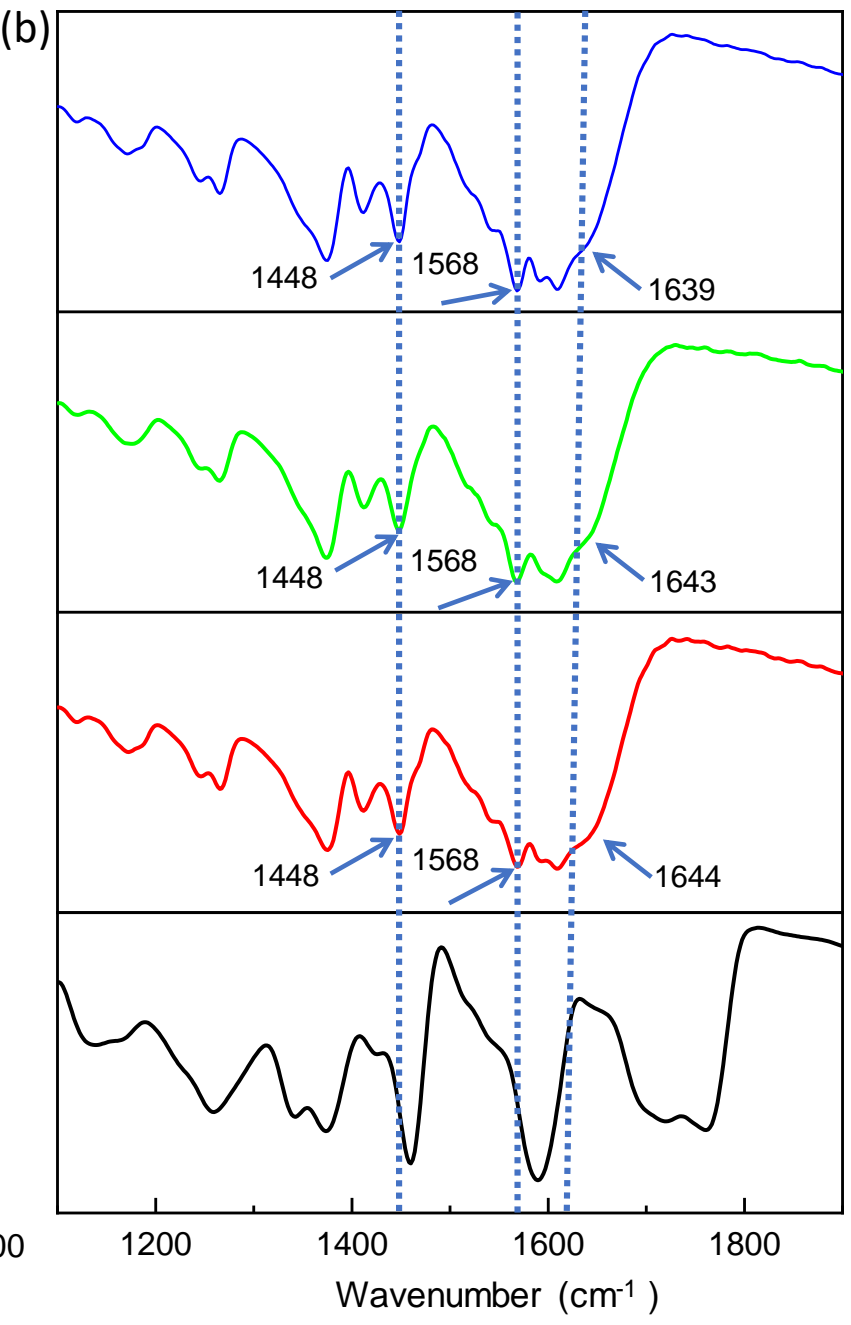

Figure S4. (a) Comparative FT-IR spectra of polyEu-Li ${ }^{+}$, polyEu-Na ${ }^{+}$, polyEu-K ${ }^{+}$, and $\mathbf{L}$ and (b) their expanded spectra in the range of $1100-1900 \mathrm{~cm}^{-1}$.

Note: In figure indicating stretching frequencies $\left(1448,1568\right.$, and $1643 \mathrm{~cm}^{-1}$ ) are responsible for the Eu-O-C bond formation. This result confirms the formation of europium complex. 


\section{XPS Spectra of polymers}

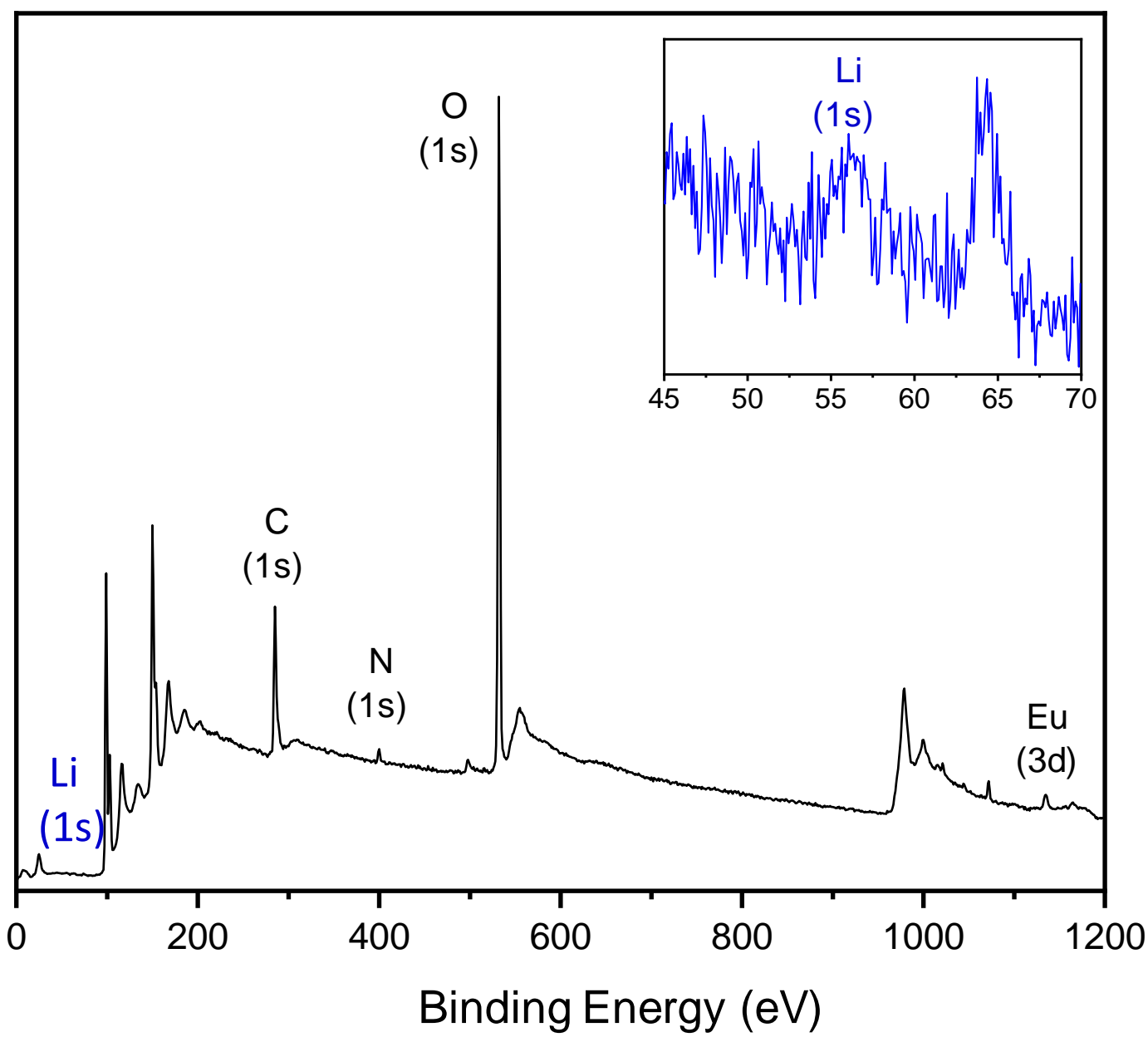

Figure S5. XPS spectrum of polyEu-Li ${ }^{+}$ 


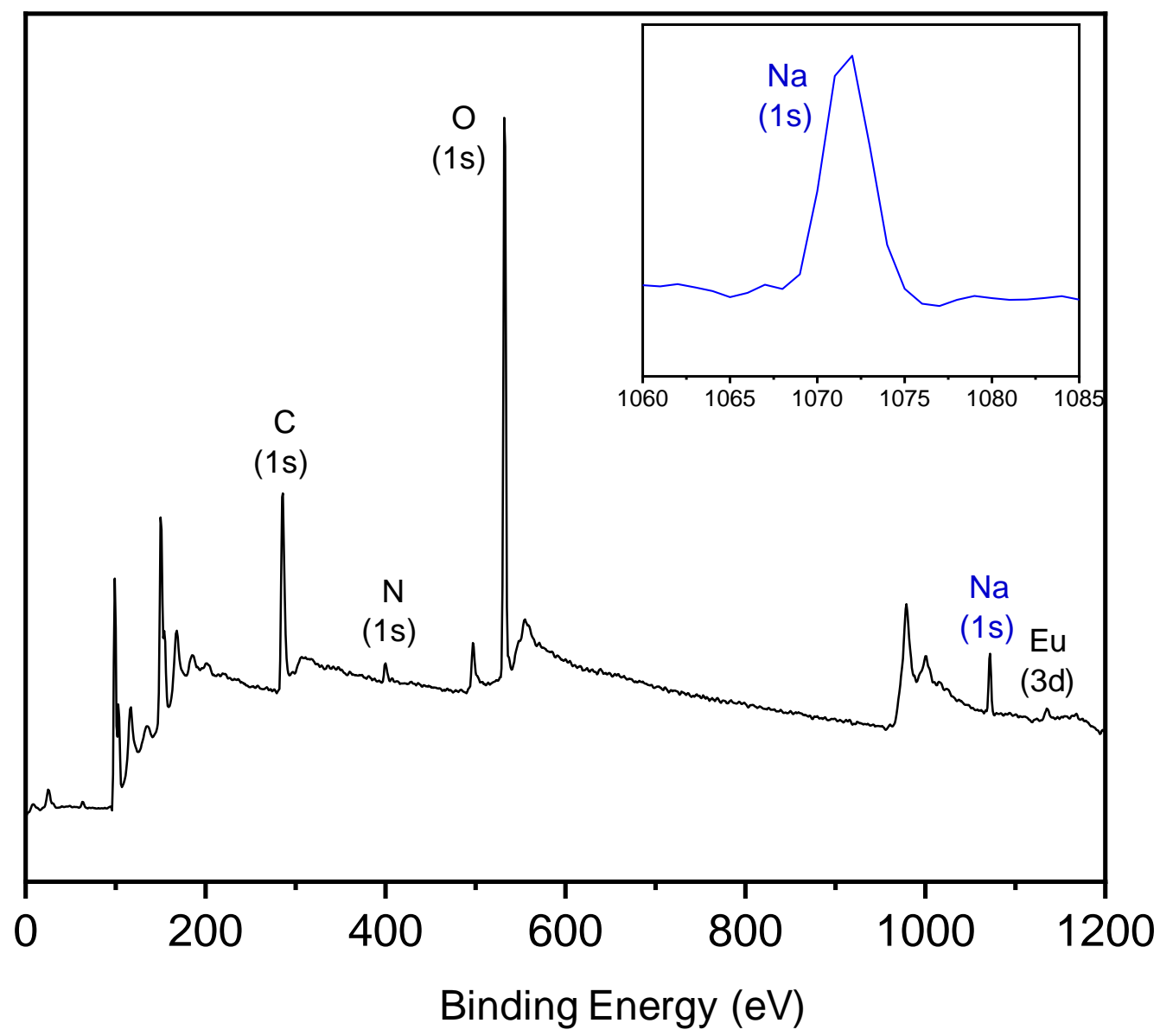

Figure S6. XPS spectrum of polyEu-Na ${ }^{+}$ 


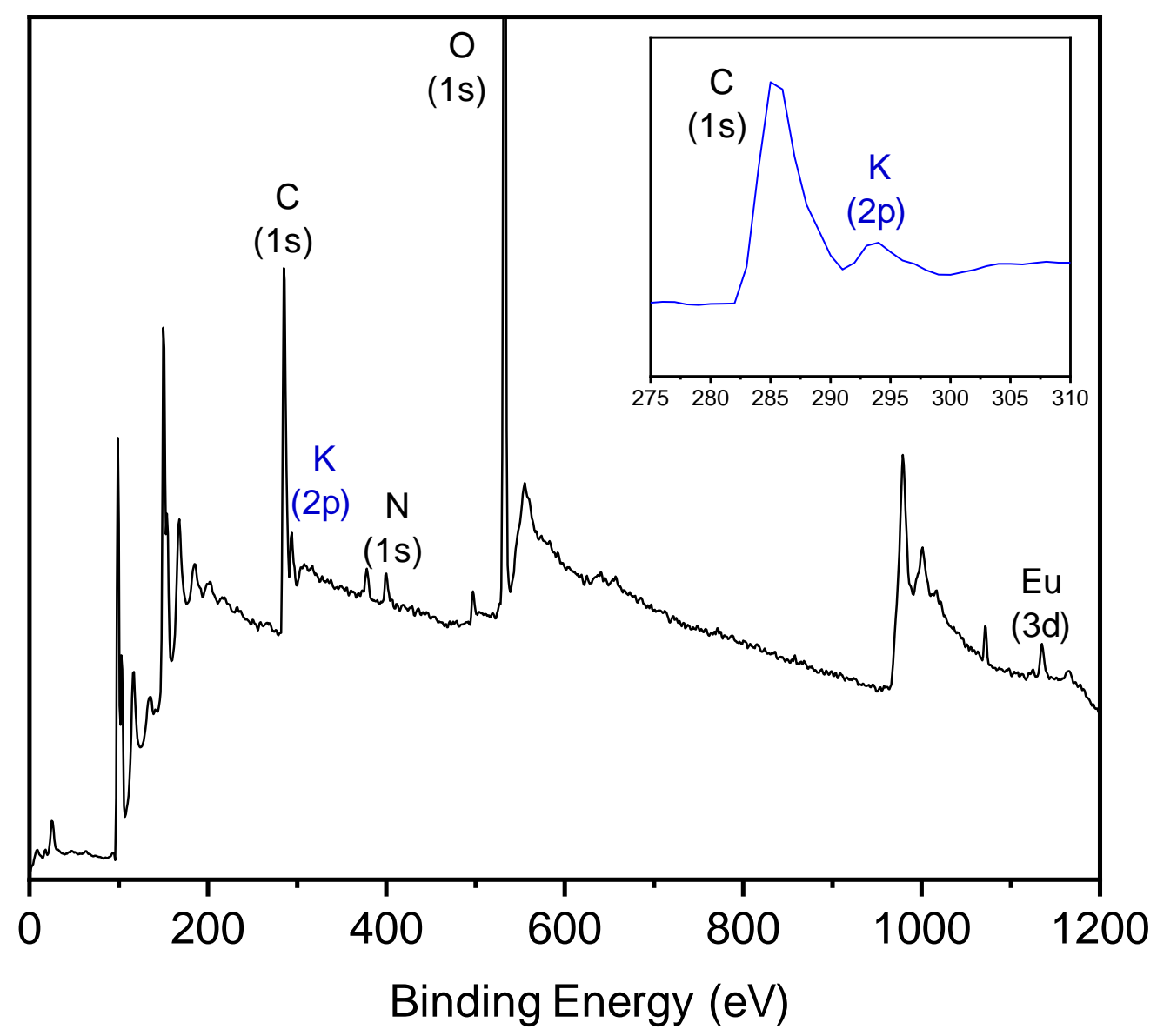

Figure S7. XPS spectrum of polyEu-K ${ }^{+}$ 
3. Thermogravimetric analysis of polymers before and after the impedance measurements
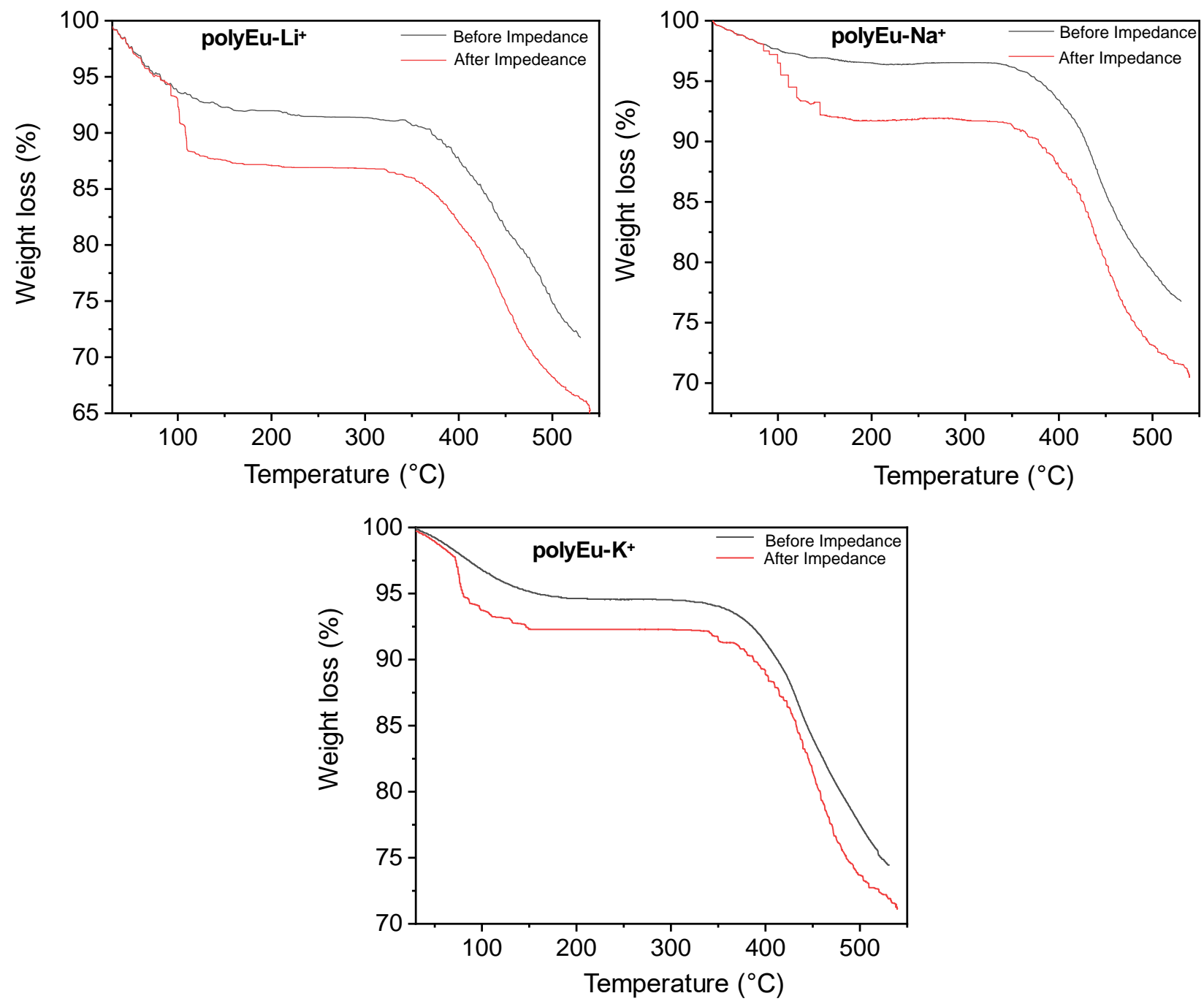

Figure S8. TGA curves of polymers 


\section{Powder -XRD Study of polymers and ligand molecule}
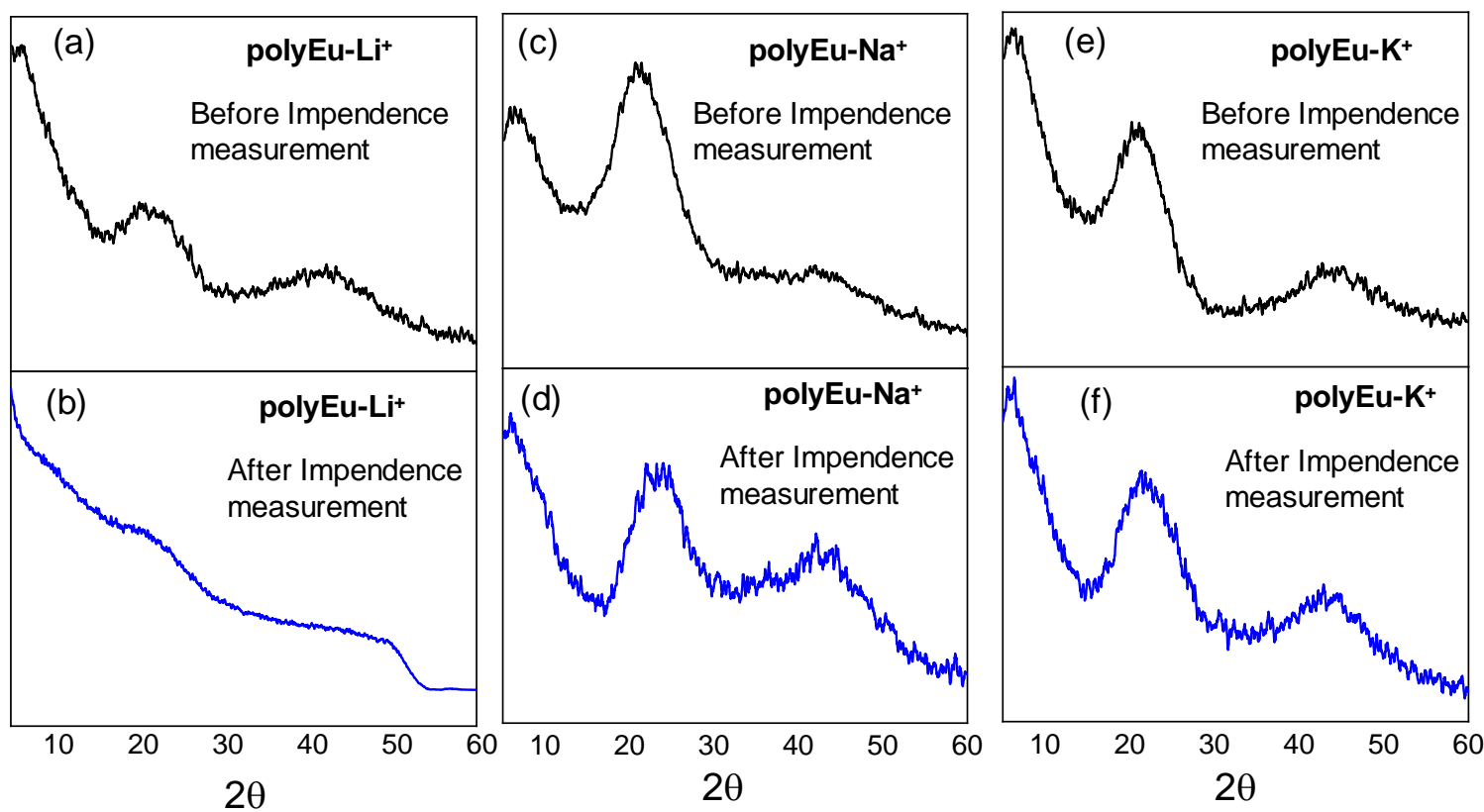

Figure S9. Powder-XRD spectra (before and after the impendence measurement) of (a,b) polyEu-Li ${ }^{+},(\mathrm{c}, \mathrm{d})$ polyEu-Na ${ }^{+}$, (e,f) polyEu-K ${ }^{+}$. 


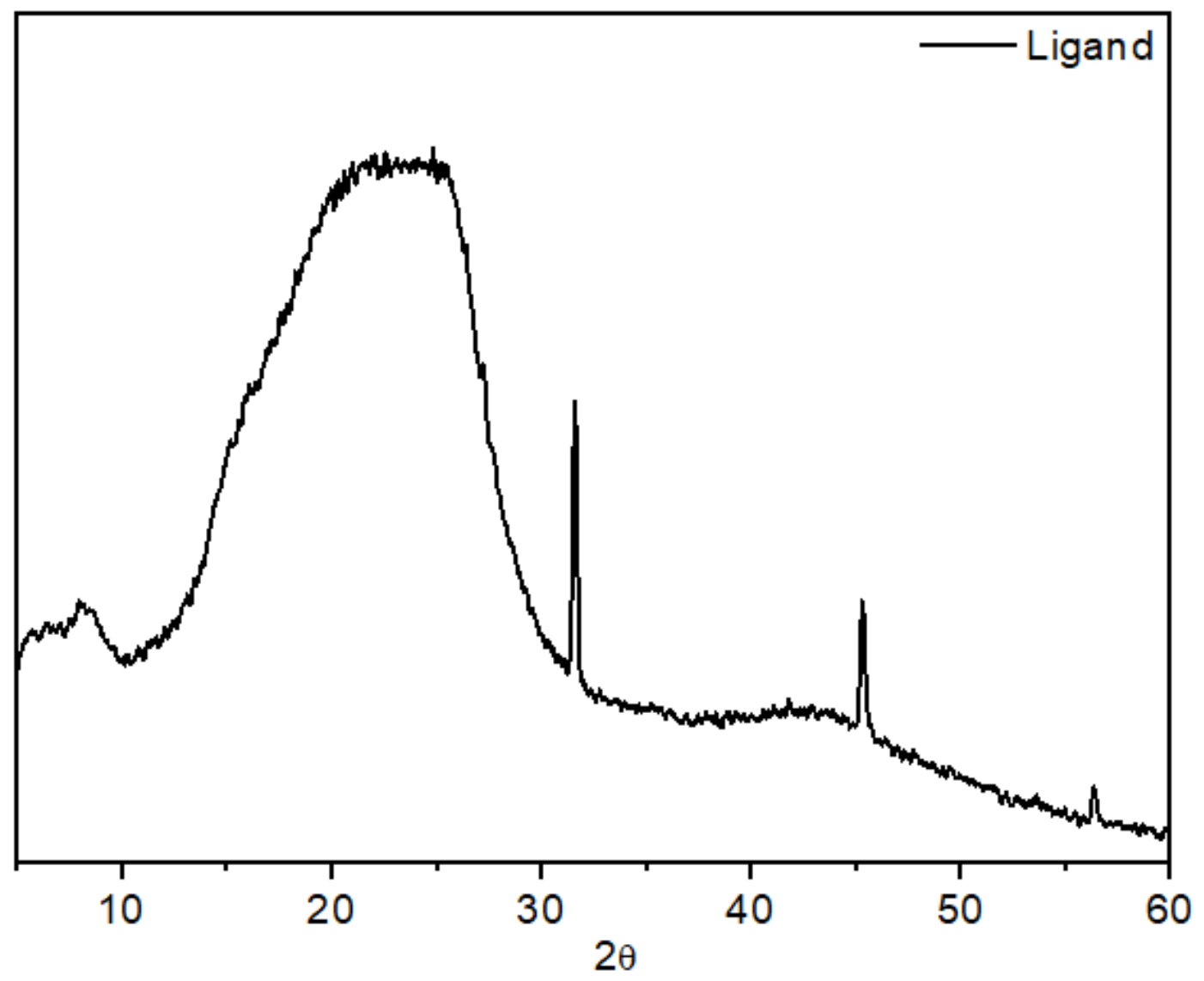

Figure S10. Powder-XRD spectra of ligand molecule 


\section{Impedance measurement of polymers using in-plane experimental method}
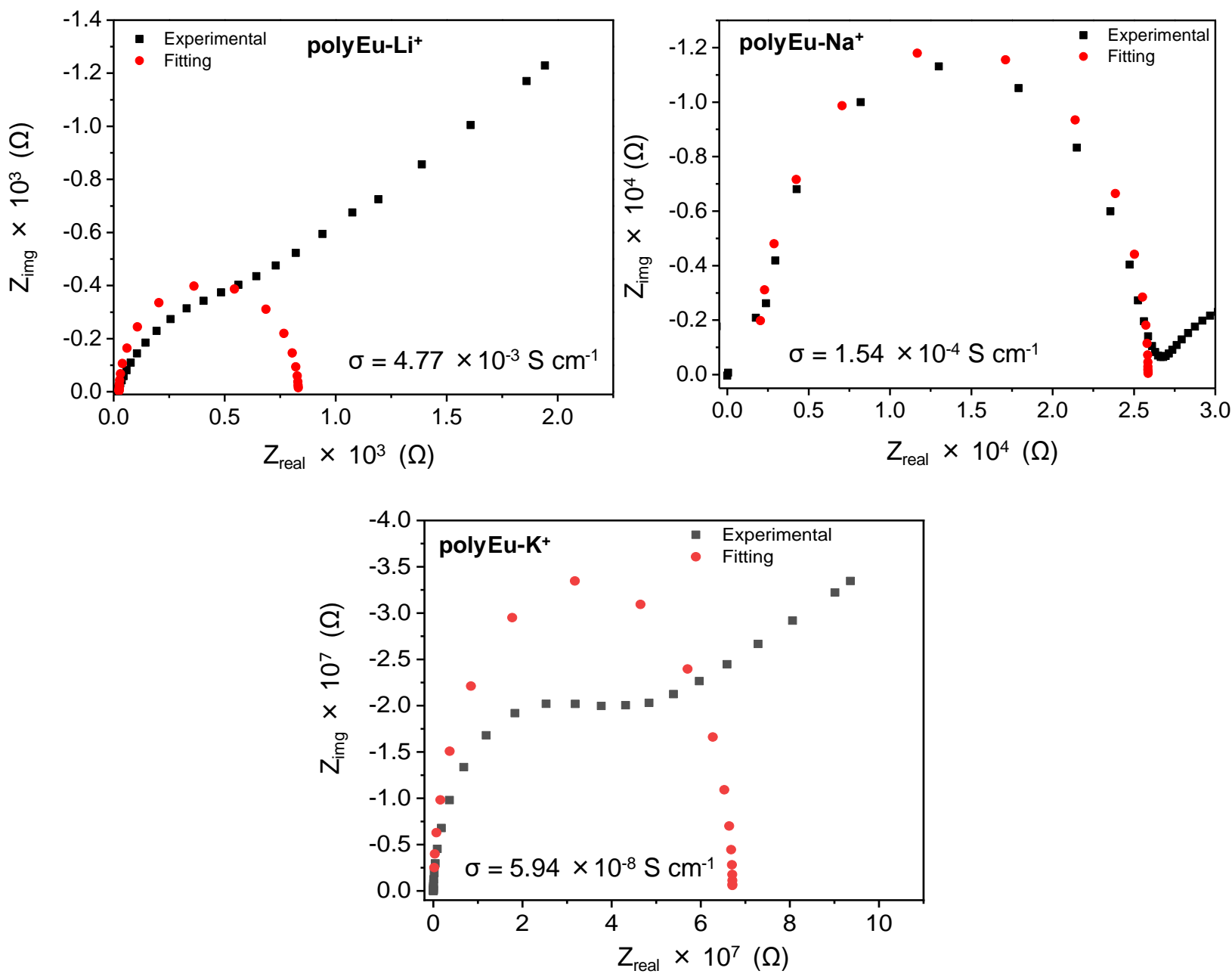

Figure S11. Nyquist plots for polymers at $25{ }^{\circ} \mathrm{C}$ temperature and $95 \% \mathrm{RH}$

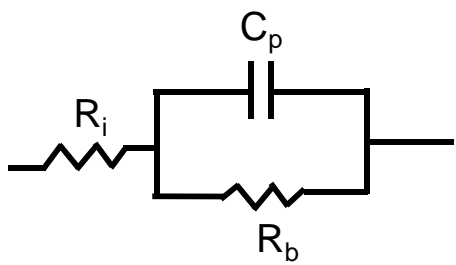

This is the equivalent circuit model used for through for fitting the experimental data of In-plane and thick film measurement. 

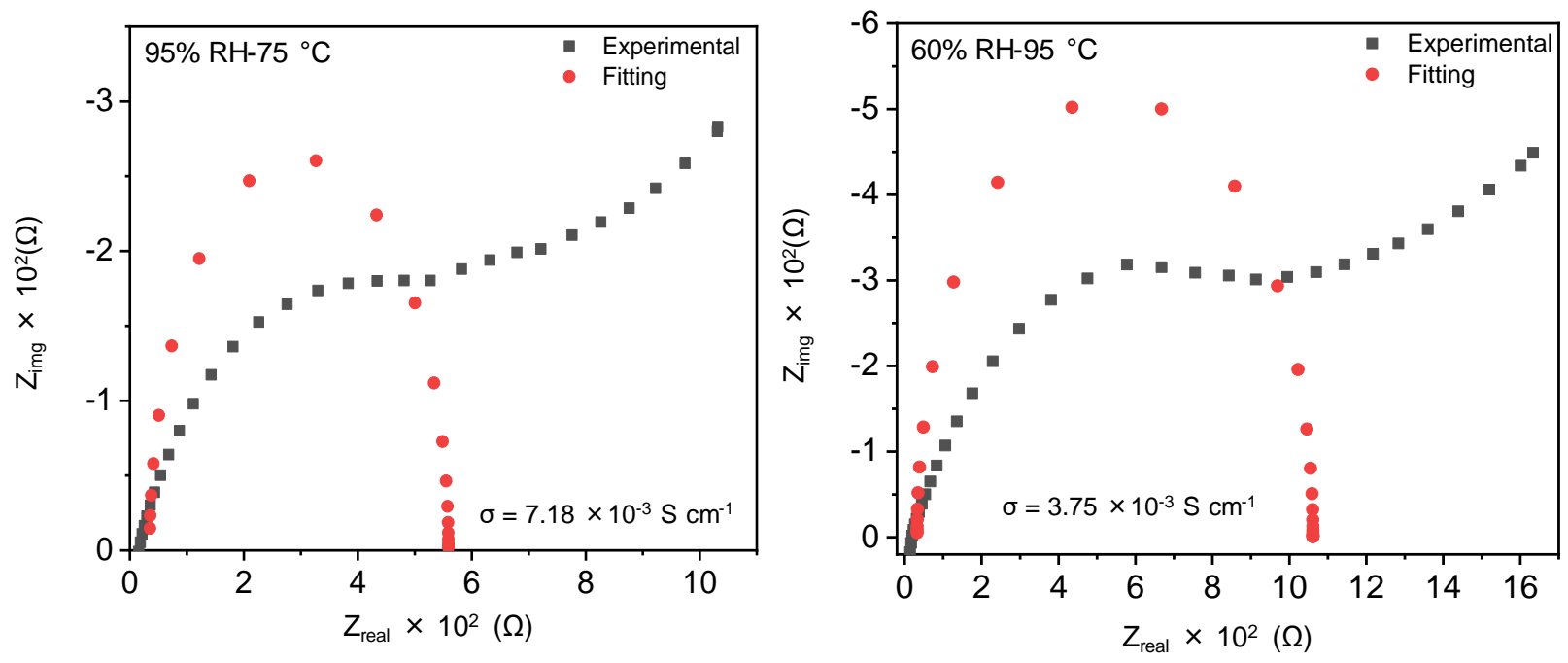

Figure S12. Nyquist plots for polyEu-Li ${ }^{+}$at at $95 \% \mathrm{RH}$ and $75{ }^{\circ} \mathrm{C}$ and $60 \% \mathrm{RH}$ and $95^{\circ} \mathrm{C}$ 


\section{Conductivity electrode (Platinum) used for in-plane experiment}

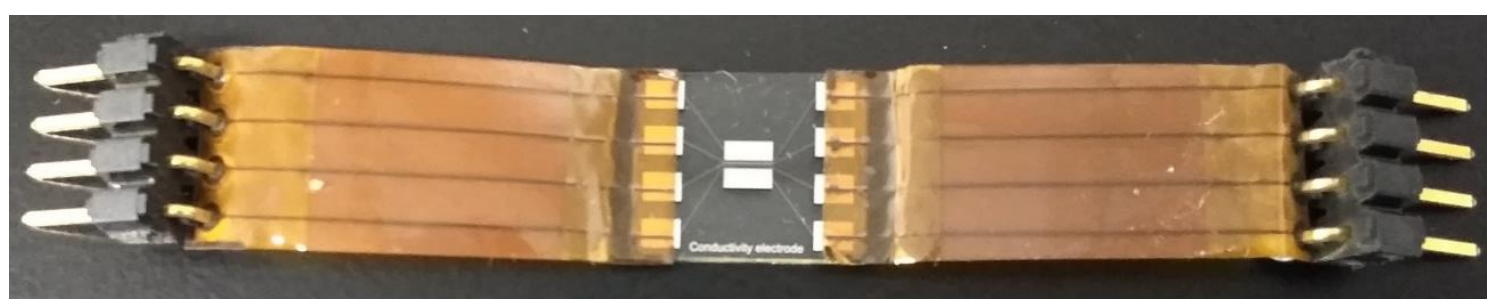

Figure S13. Photo-graph of platinum-based conductivity electrode

Note: This the electrode made by the deposition of platinum on a quartz glass substrate. The conductivity electrode was purchased from BAS Co., Ltd, Japan.

Product code -011316 Conductivity electrode

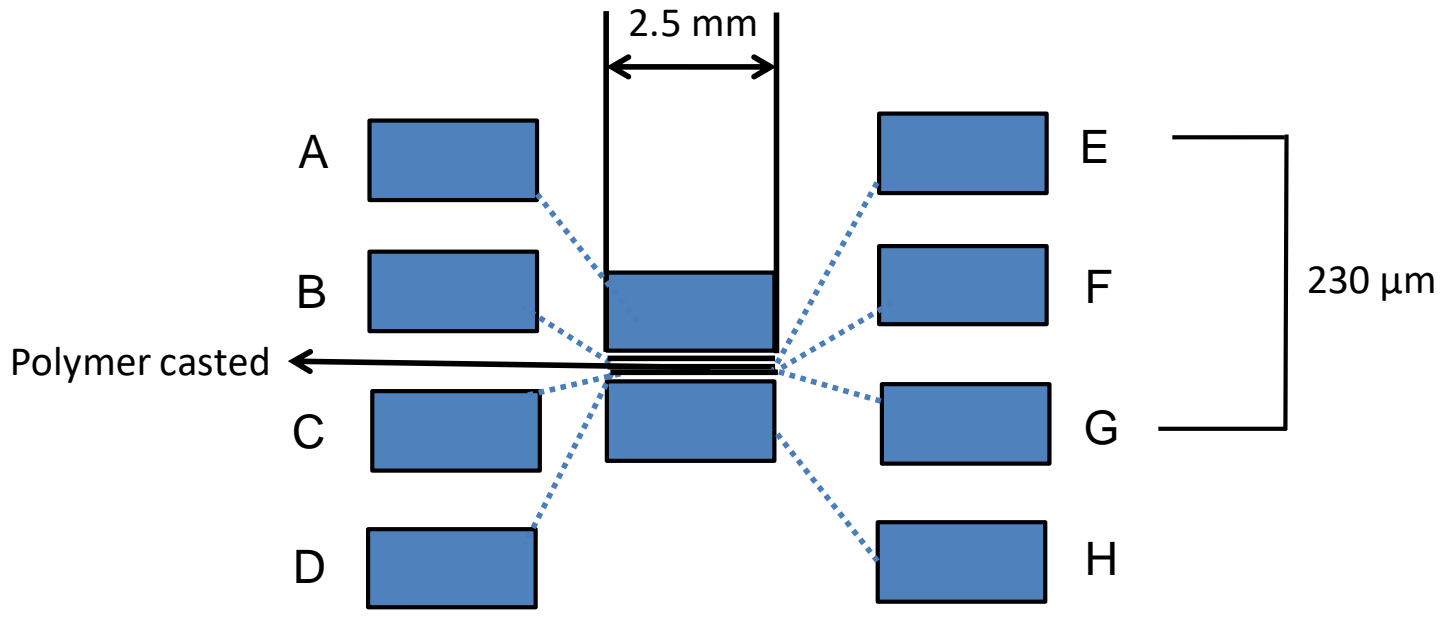

Figure S14. Schematic diagram of conductivity electrode for proton conductivity measurement of the sample- In-plane method (A-H in the figure are the terminal electrodes)

In-plane experiment, the proton conductivity was calculated using the following equation

$$
\sigma=\mathrm{d} / \mathrm{RA}
$$

Here $\mathrm{d}$ is the distance between the inner electrode pair (we have connected the $\mathrm{E}$ and $\mathrm{G}$ electrodes $(\mathrm{d}=230 \mu \mathrm{m})$

$\mathrm{A}$ is the cross-sectional current carrying-area. Here $\mathrm{A}$ is the $2.5 \mathrm{~mm} \times 230 \mu \mathrm{m}, \mathrm{R}$ was obtained from the Nyquist plot. 
7. Impedance measurement of polyEu- $\mathrm{Li}^{+}$using the thick pellet.
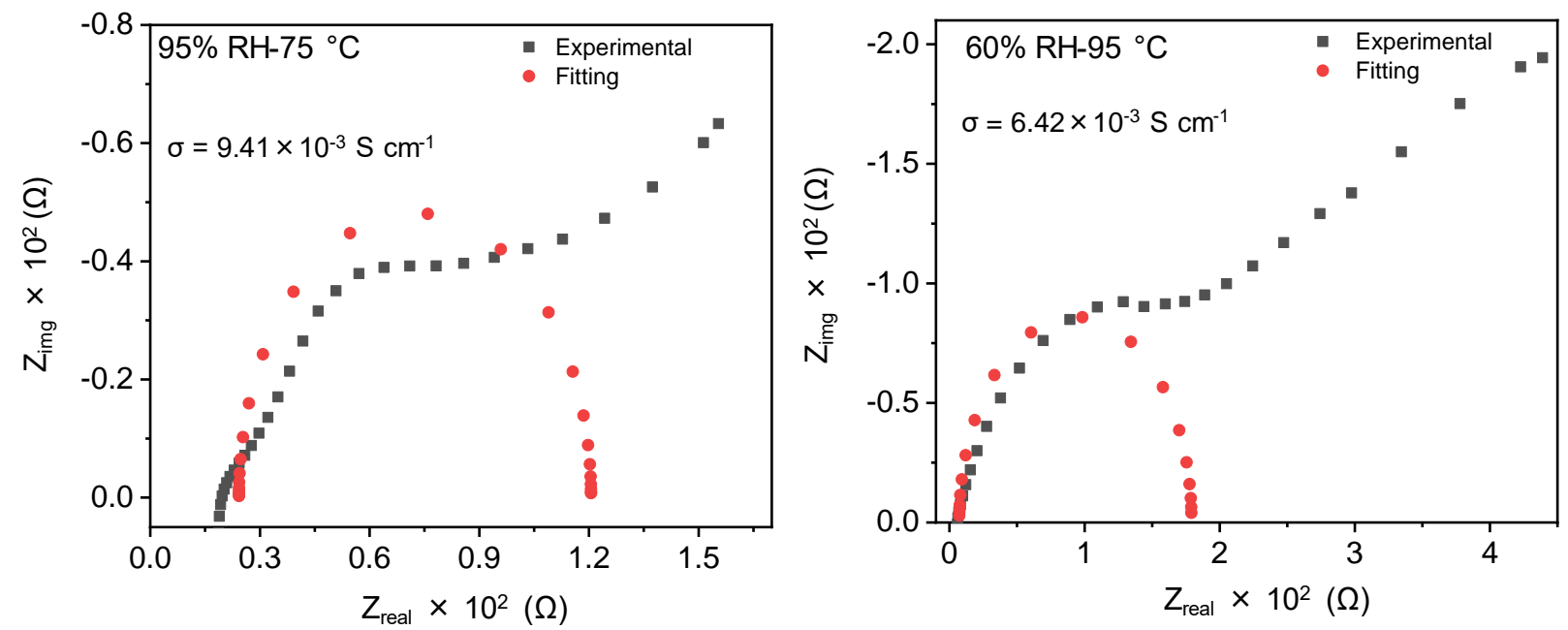

Figure S15. Nyquist plots for polyEu $-\mathbf{L i}^{+}$at $95 \% \mathrm{RH}$ and $75^{\circ} \mathrm{C}$ and $60 \% \mathrm{RH}$ and $95^{\circ} \mathrm{C}$

Note: We have used $0.8 \mathrm{~mm}$ thick ( $3 \mathrm{~mm}$ diameter) pellet for the impedance measurement 
8. Impedance measurement of polymers at different humidity condition

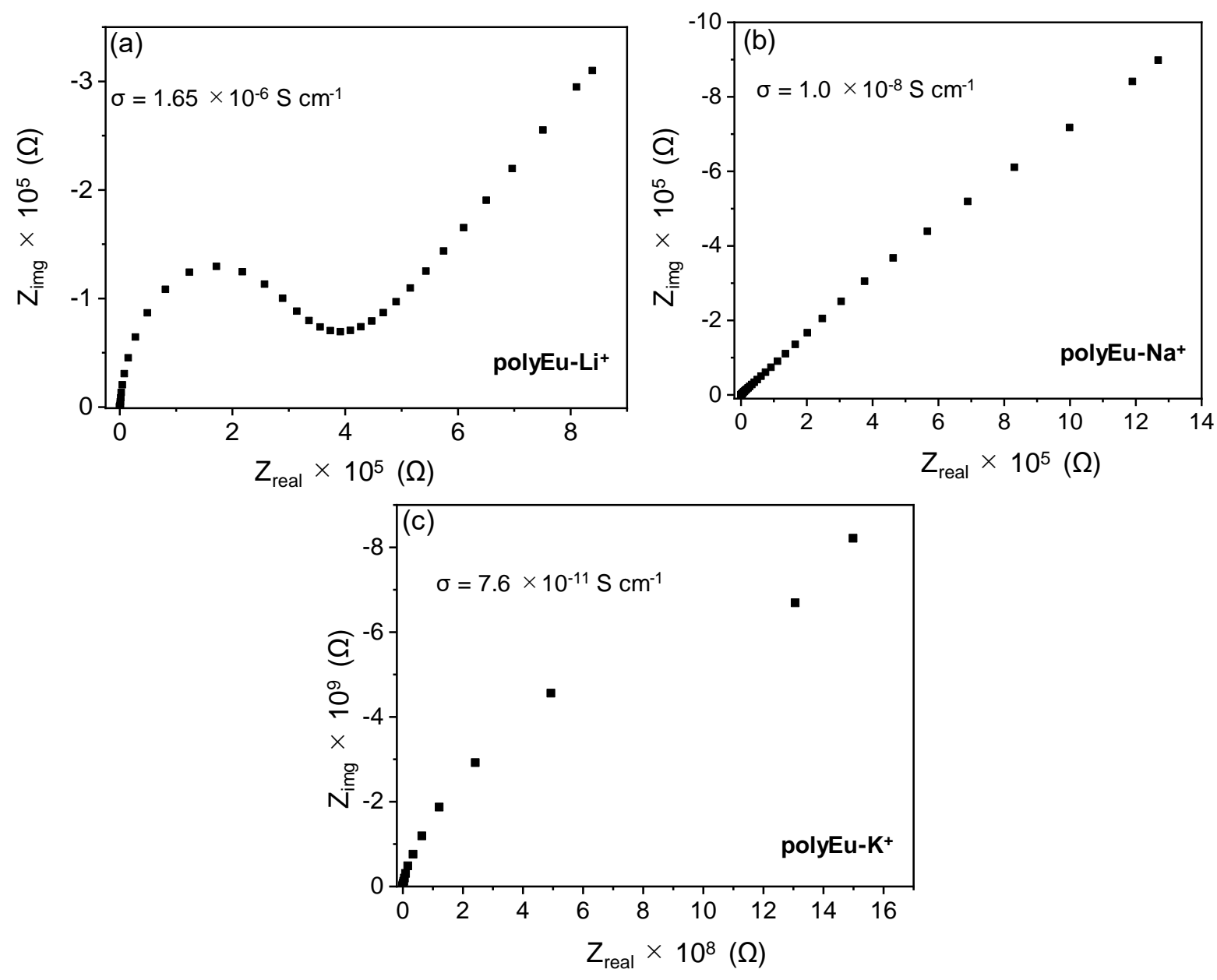

Figure S16. Nyquist plots for polymers at $25^{\circ} \mathrm{C}$ temperature and $30 \% \mathrm{RH}$ (a) polyEu-Li ${ }^{+}$ (b) polyEu-Na+ ${ }^{+}$c) polyEu-K ${ }^{+}$ 

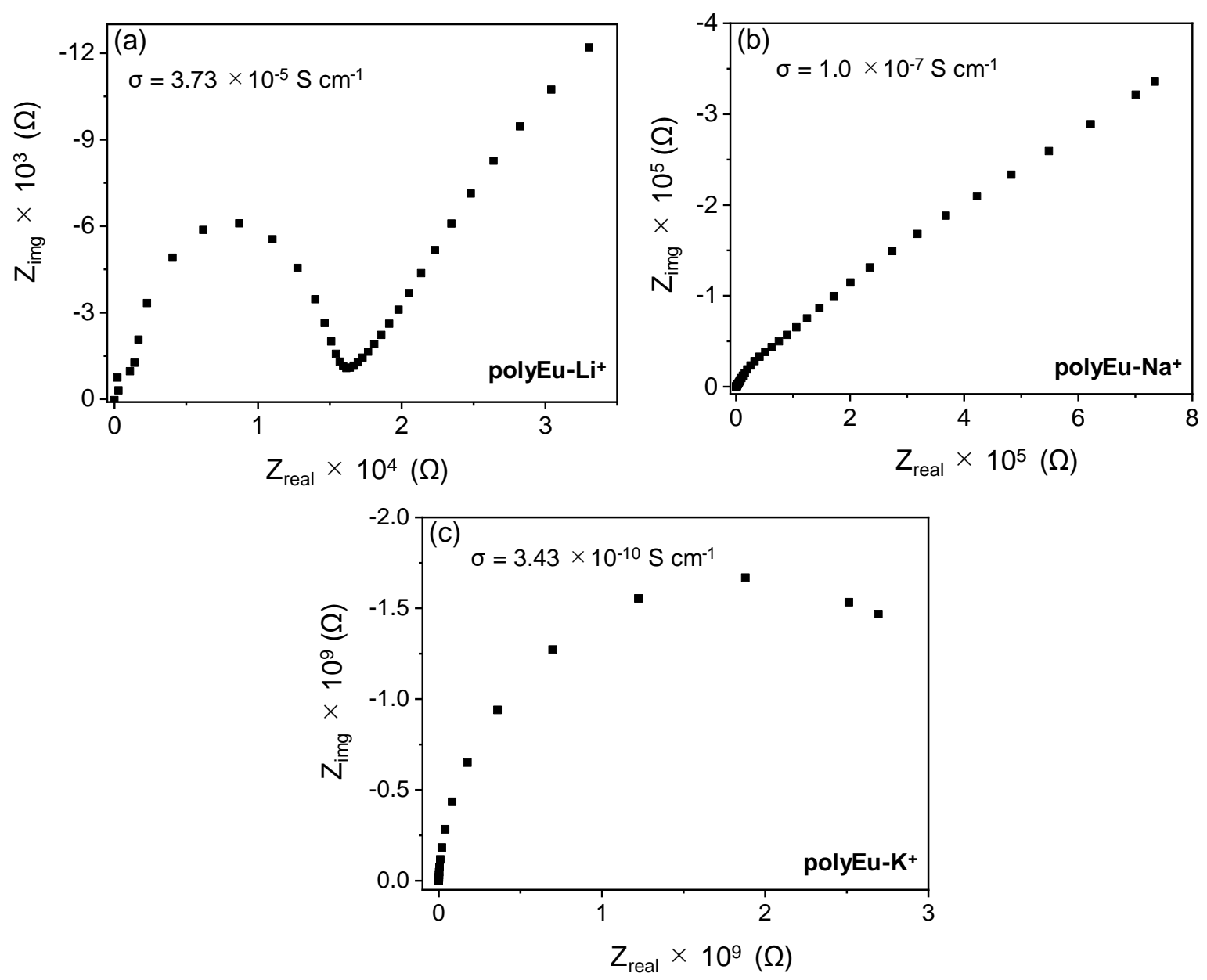

Figure S17. Nyquist plots for polymers at $25{ }^{\circ} \mathrm{C}$ temperature and $50 \%$ RH (a) polyEu-Li ${ }^{+}$ (b) polyEu-Na+ ${ }^{+}$(c) polyEu-K ${ }^{+}$ 

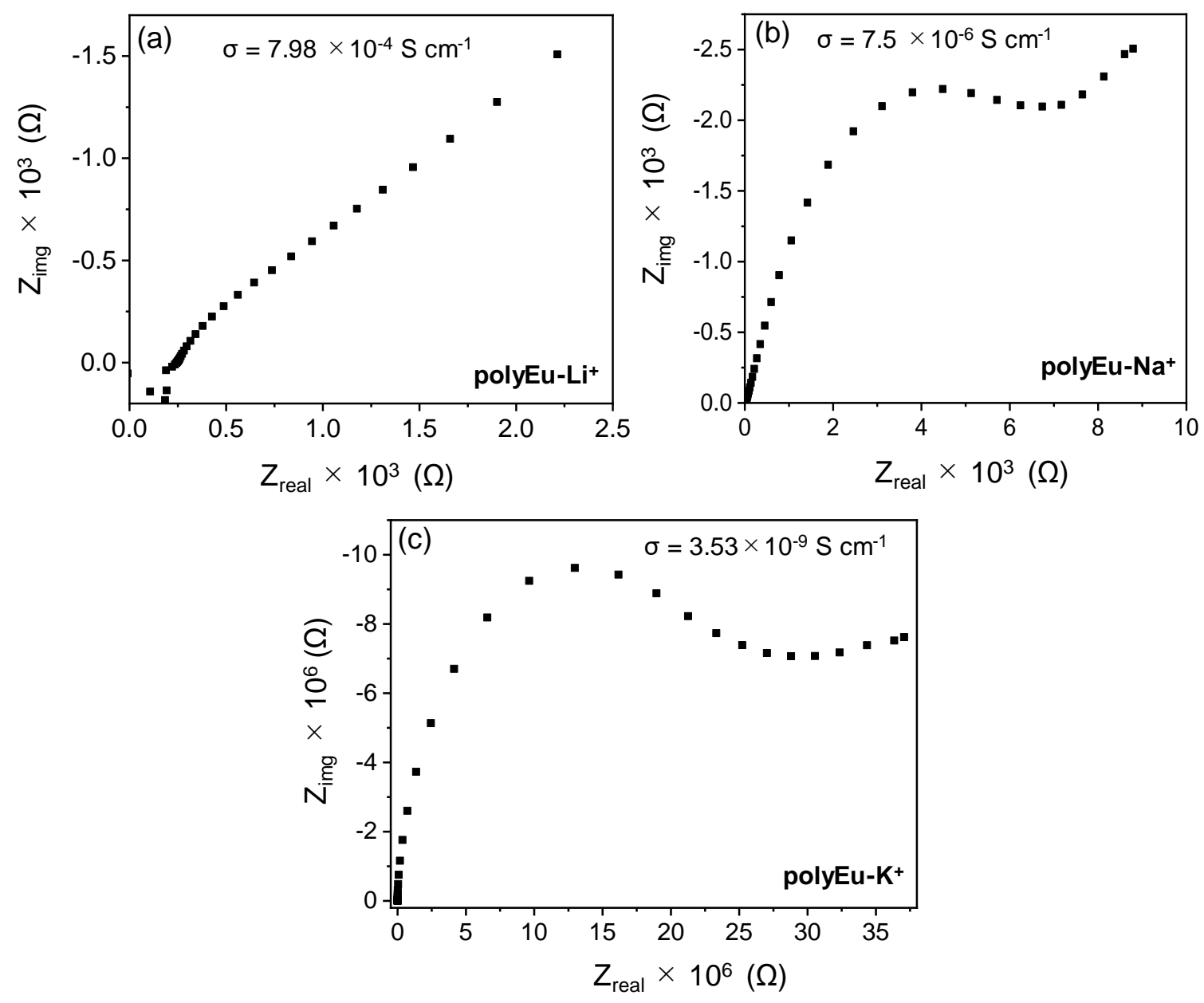

Figure S18. Nyquist plots for polymers at $25{ }^{\circ} \mathrm{C}$ temperature and $70 \% \mathrm{RH}$ (a) polyEu-Li ${ }^{+}$ (b) polyEu-Na+ ${ }^{+}$(c) polyEu-K ${ }^{+}$ 
9. Log conductivity vs log frequency plots of polymers for through plane, in-plane and thick films data.

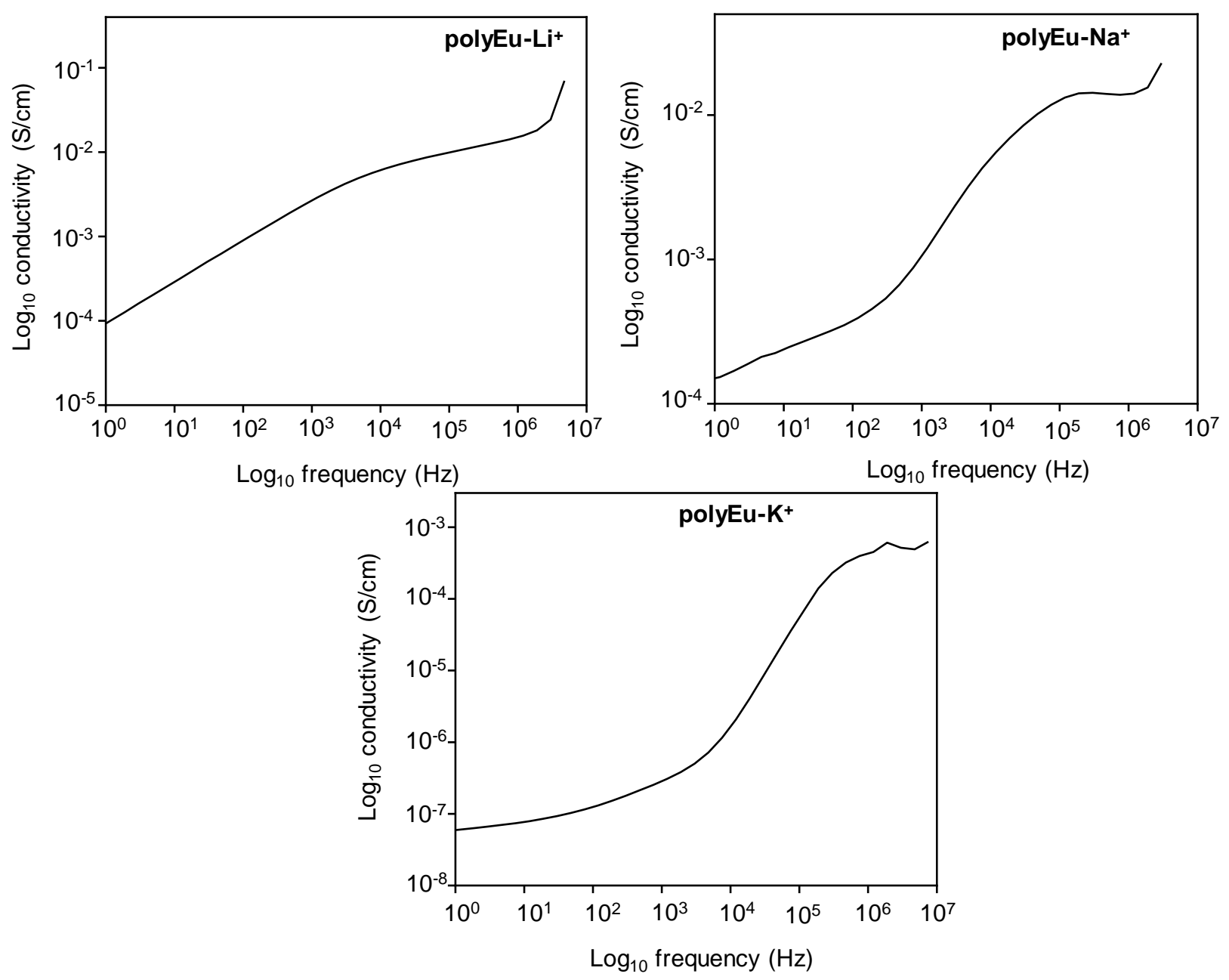

Figure S19. Bode plots (Log conductivity vs log frequency) of Figure $3 a$ and b (Through plane data) 

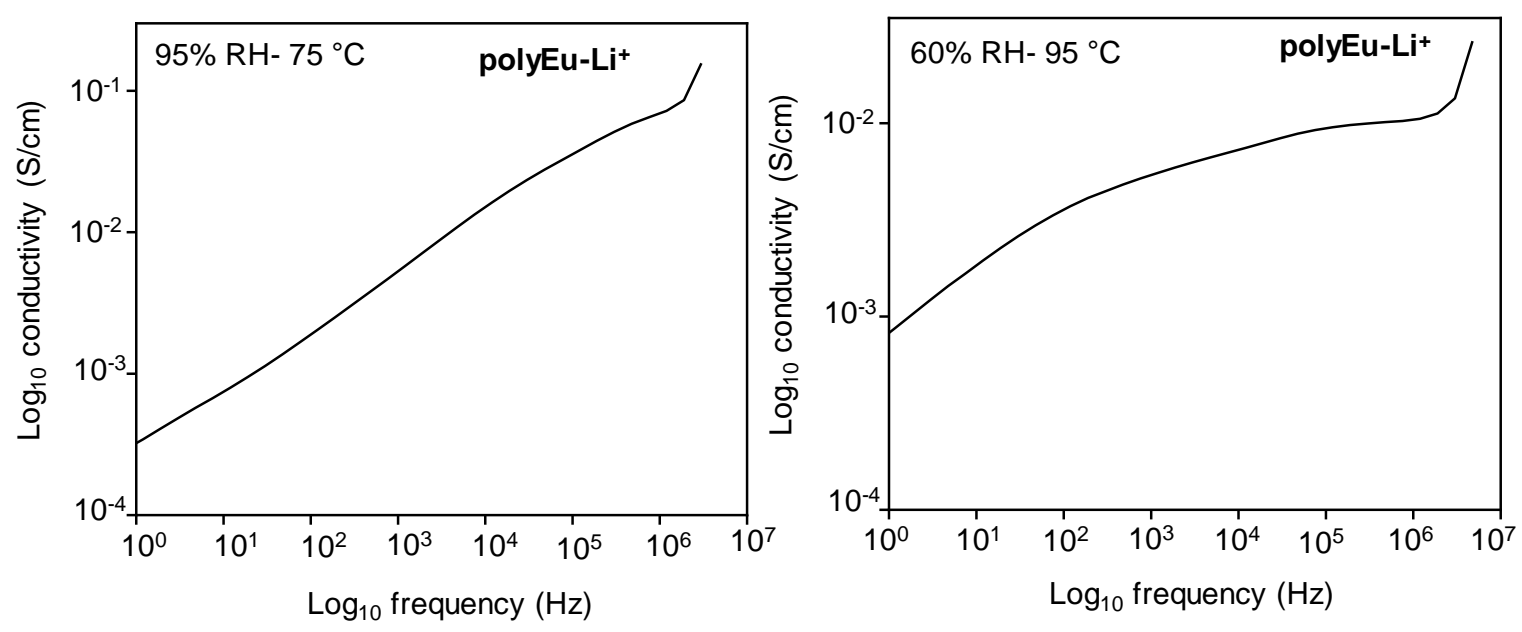

Figure S20. Bode plots (Log conductivity vs $\log$ frequency) of Figure $4 a$ and $b$ (Through plane data) 

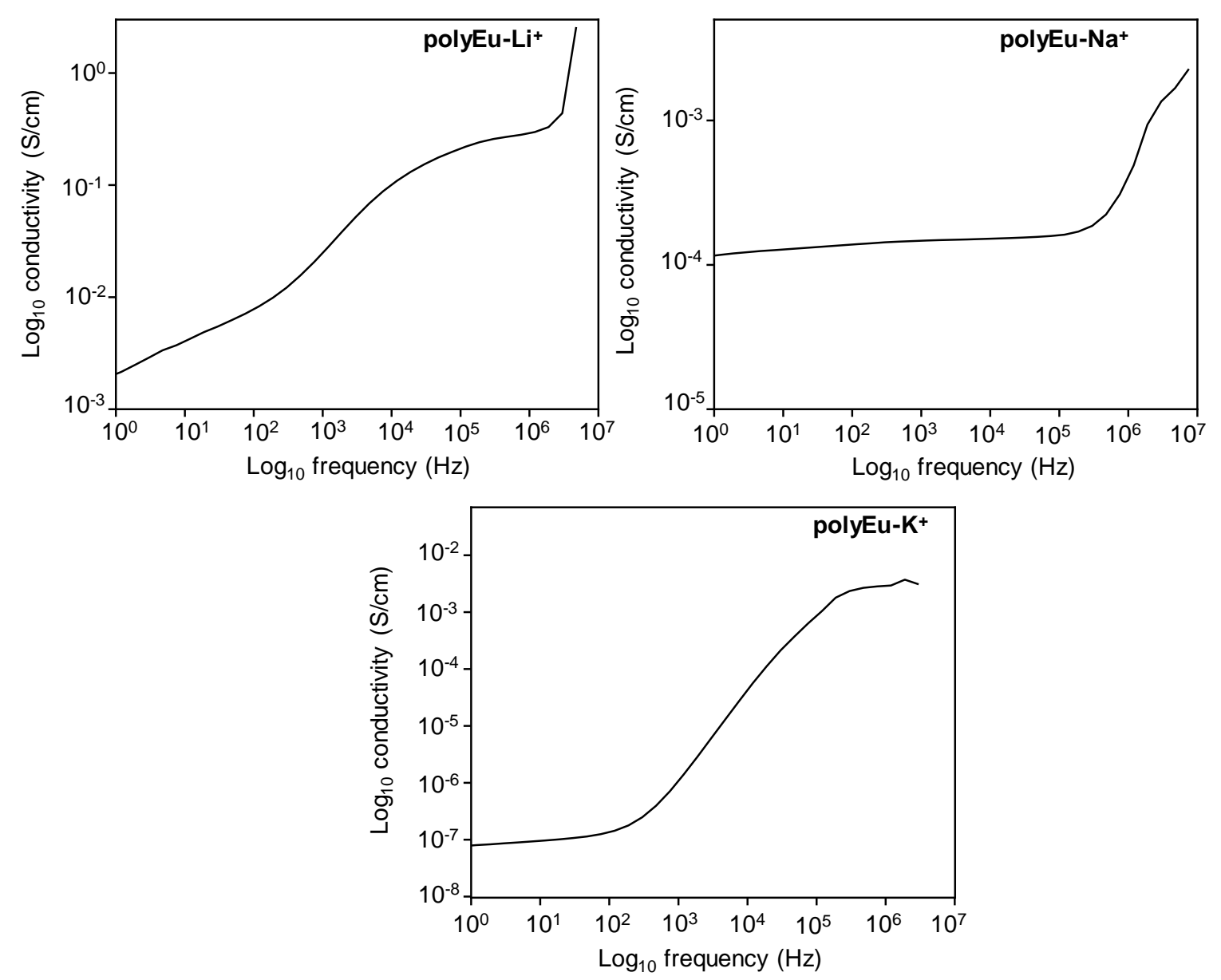

Figure S21. Bode plots (Log conductivity vs log frequency) of experimental data of Figure S11 ( In-plane measurement). 

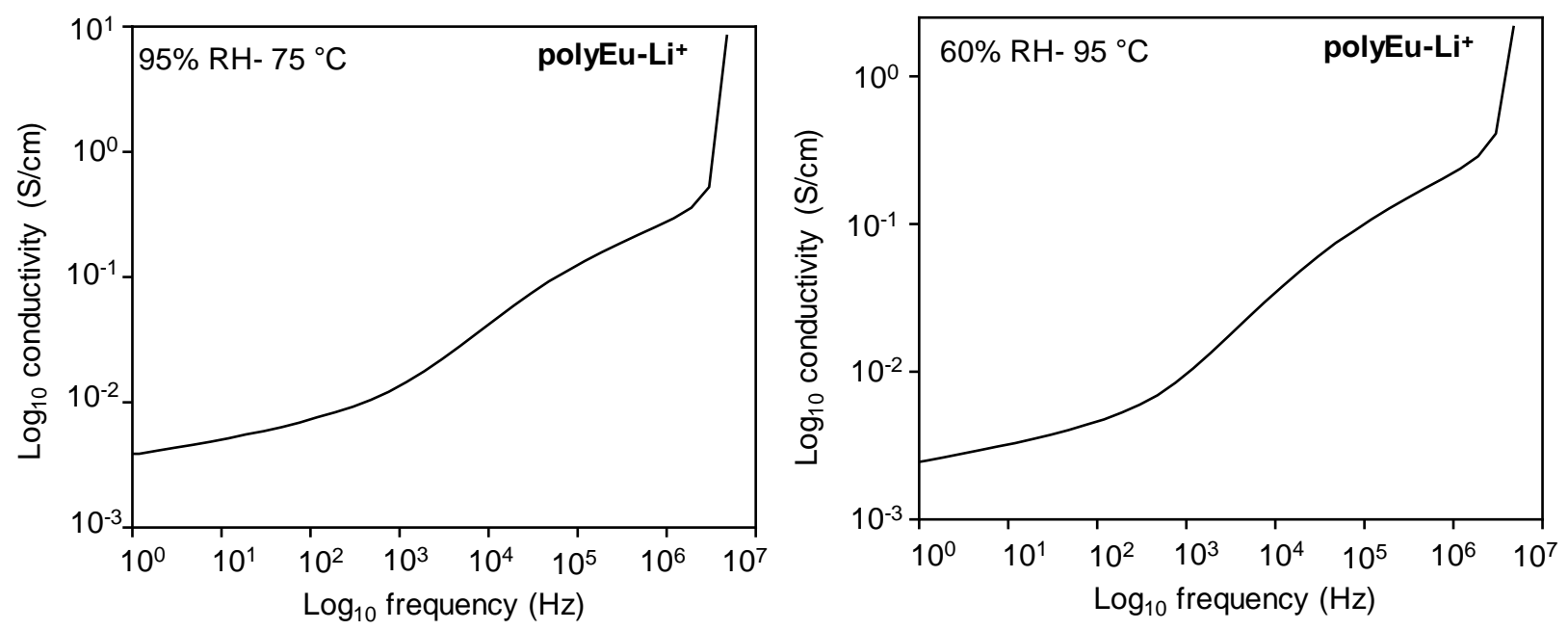

Figure S22. Bode plots (Log conductivity vs log frequency) of experimental data of Figure S12 (In-plane measurement). 

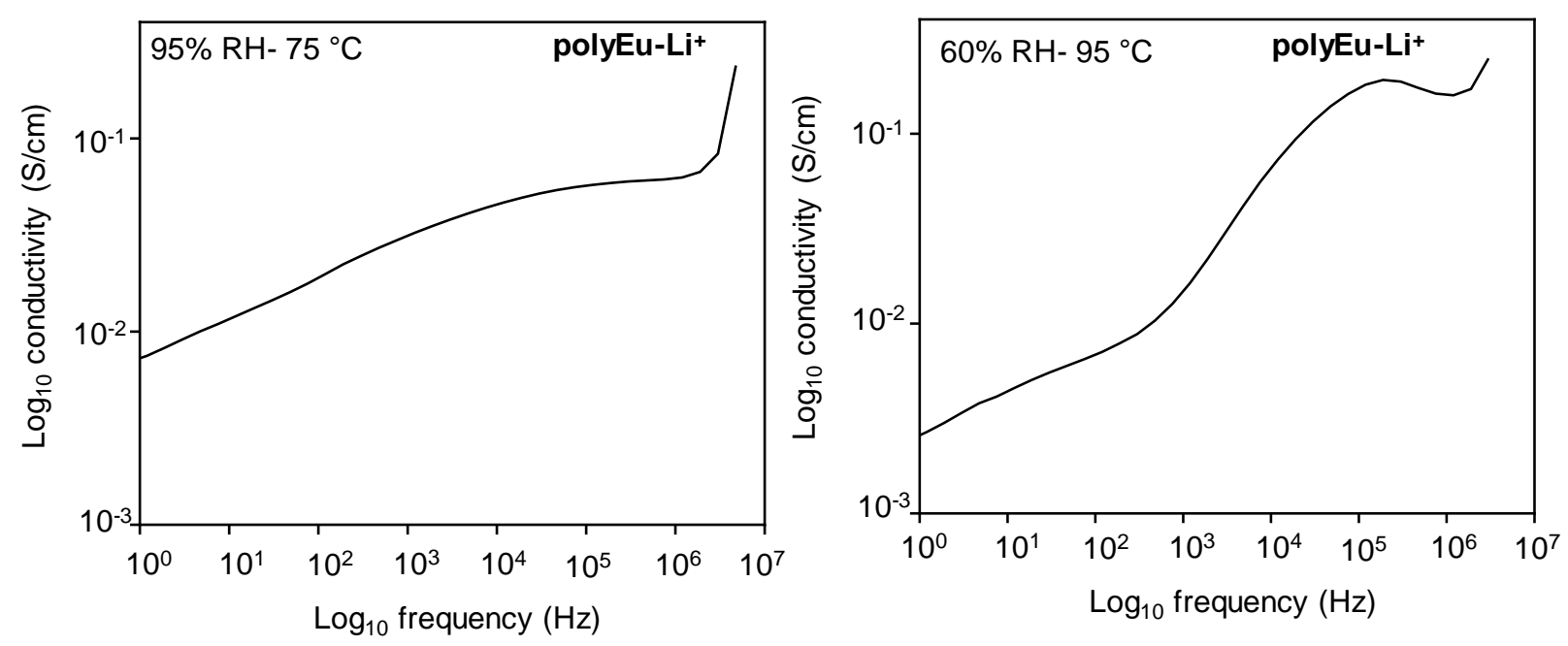

Figure S23. Bode plots (Log conductivity vs log frequency) of experimental data of Figure S15 (Thick film measurement). 
10. PL Spectra of polymers at ambient and at $95 \%$ RH conditions
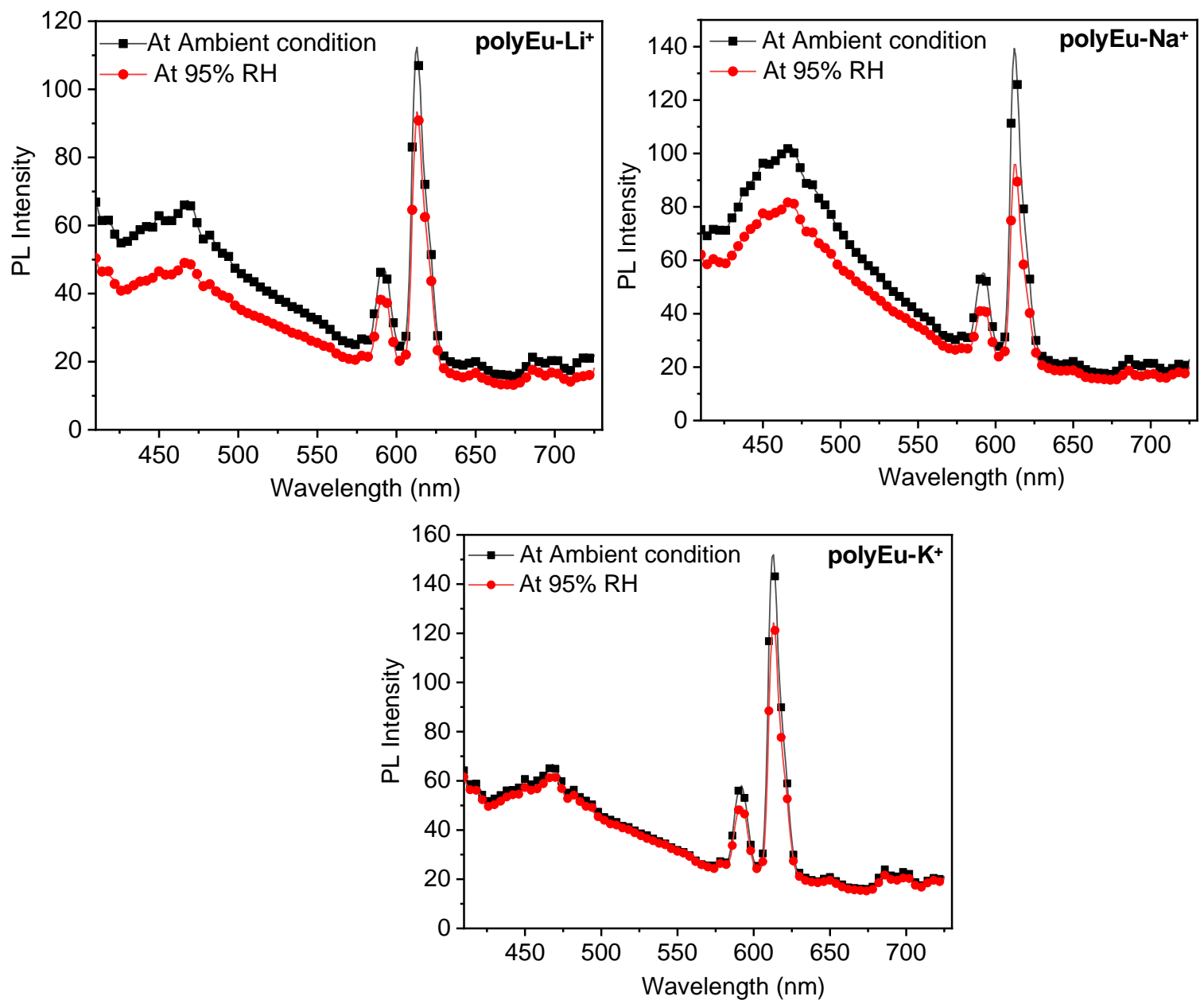

Figure S24. Comparative PL spectra of polymers at ambient and 95\% RH conditions. 
11. Through -plane and in-plane proton conductivities of polymers at $95 \% \mathrm{RH}$ and $25{ }^{\circ} \mathrm{C}$

Table S1. Comparative proton conductivities of polymers using different experiment setup at $95 \% \mathrm{RH}$ and $25{ }^{\circ} \mathrm{C}$

\begin{tabular}{|c|c|c|c|}
\hline Method & polyEu-Li $^{+}$ & polyEu-Na $^{+}$ & polyEu-K $^{+}$ \\
\hline Through plane & $6.60 \times 10^{-3}$ & $3.90 \times 10^{-4}$ & $7.80 \times 10^{-8}$ \\
\hline In-plane & $4.77 \times 10^{-3}$ & $1.54 \times 10^{-4}$ & $5.90 \times 10^{-8}$ \\
\hline
\end{tabular}


12. Comparative proton conductivities of polymers at different humidity

Table S2. Comparative proton conductivities of polymers at different humidity and $25{ }^{\circ} \mathrm{C}$

\begin{tabular}{|c|c|c|c|}
\hline \% RH & polyEu-Li $^{+}$ & polyEu-Na $^{+}$ & polyEu-K $^{+}$ \\
\hline 30 & $1.65 \times 10^{-6}$ & $1.01 \times 10^{-8}$ & $7.60 \times 10^{-11}$ \\
\hline 40 & $4.27 \times 10^{-6}$ & $6.02 \times 10^{-8}$ & $1.01 \times 10^{-10}$ \\
\hline 50 & $3.73 \times 10^{-5}$ & $1.10 \times 10^{-7}$ & $3.43 \times 10^{-10}$ \\
\hline 60 & $4.61 \times 10^{-4}$ & $9.01 \times 10^{-7}$ & $8.01 \times 10^{-10}$ \\
\hline 70 & $7.98 \times 10^{-4}$ & $7.50 \times 10^{-6}$ & $3.53 \times 10^{-9}$ \\
\hline 80 & $9.55 \times 10^{-4}$ & $9.50 \times 10^{-6}$ & $1.76 \times 10^{-8}$ \\
\hline 90 & $2.65 \times 10^{-3}$ & $9.80 \times 10^{-5}$ & $3.01 \times 10^{-8}$ \\
\hline 95 & $6.60 \times 10^{-3}$ & $3.90 \times 10^{-4}$ & $7.80 \times 10^{-8}$ \\
\hline
\end{tabular}

